\title{
GENERATING FINITE COMPLETELY REDUCIBLE LINEAR GROUPS
}

\author{
L. G. KOVÁCS AND GEOFFREY R. ROBINSON
}

(Communicated by Warren J. Wong)

\begin{abstract}
It is proved here that each finite completely reducible linear group of dimension $d$ (over an arbitrary field) can be generated by $\left\lfloor\frac{3}{2} d\right\rfloor$ elements. If a finite linear group $G$ of dimension $d$ is not completely reducible, then its characteristic is a prime, $p$ say, and the factor group of $G$ modulo the largest normal $p$-subgroup $\mathbb{O}_{p}(G)$ may be viewed as a completely reducible linear group acting on the direct sum of the composition factors of the natural module for $G$ : consequently, $G / \mathbb{O}_{p}(G)$ can still be generated by $\left\lfloor\frac{3}{2} d\right\rfloor$ elements.
\end{abstract}

\section{INTRODUCTION}

The aim of this paper is to prove the following:

Theorem. Each finite completely reducible linear group of dimension $d$ can be generated by $\left\lfloor\frac{3}{2} d\right\rfloor$ elements.

If a finite linear group $G$ of dimension $d$ is not completely reducible, then its characteristic is a prime, $p$ say, and the factor group of $G$ modulo the largest normal $p$-subgroup $\mathbb{O}_{p}(G)$ may be viewed as a completely reducible linear group acting on the direct sum of the composition factors of the natural module for $G$ : consequently, $G / \mathbb{O}_{p}(G)$ can still be generated by $\left\lfloor\frac{3}{2} d\right\rfloor$ elements.

By a linear group $G$ we mean a group of nonsingular linear transformations on a finite dimensional vector space $V$ over a (commutative) field $\mathbb{F}$; the dimension of $G$ is the dimension of $V$. As usual, for any real number $x$ we denote by $\lfloor x\rfloor$ and $\lceil x\rceil$ the integers defined by $\lfloor x\rfloor \leq x<\lfloor x\rfloor+1$ and $\lceil x\rceil-1<x \leq\lceil x\rceil$.

The case of a group whose order is prime to the characteristic of the field can be dealt with relatively easily. Isaacs had shown [9] that each finite completely reducible linear $p$-group of dimension $d$ can be generated by $\left\lfloor\frac{3}{2} d\right\rfloor$ elements. Lucchini [12] and Guralnick [6] have recently proved that any (abstract) finite group can be generated by $n+1$ elements provided each of its Sylow subgroups

Received by the editors February 23, 1990 and, in revised form, April 8, 1990.

1980 Mathematics Subject Classification (1985 Revision). Primary 20C15, 20 C20.

Part of this work was done while the second author held a Visiting Fellowship at the Australian National University. 
can be generated by $n$ elements. With a little care, one can combine these two results (and Maschke's theorem) to get what we want.

This plan cannot possibly work without the coprimality assumption: for example, the 2-dimensional irreducible linear groups $S L\left(2, p^{n}\right)$ have Sylow subgroups which need $n$ generators. Instead, we show that in any finite completely reducible linear group of prime characteristic $p$, the difficulties arising from possibly large Sylow $p$-subgroups can be contained within the components (that is, the quasisimple subnormal subgroups) of $G$ : then these difficulties can be overcome by using that all quasisimple groups are 2-generator groups.

For the latter fact, and for information on outer automorphism groups of simple groups, we depend directly on the classification of finite simple groups. Use of the work of Guralnick and Lucchini already made us indirectly dependent on that. It will be convenient to exploit the 2-generator nature of simple groups via an easy variant (which we take for granted) of Lemma 2 of Wiegold [14]: any direct product of $r$ nonabelian finite simple groups can be generated by $2+\left\lceil\log _{60} r\right\rceil$ elements.

If $\mathbb{F}$ is any field which has an element of multiplicative order 4 , then $G L(2, \mathbb{F})$ has an irreducible subgroup of order 16 which needs 3 generators. The direct sum of $m$ copies of this group gives a $2 m$-dimensional linear group which cannot be generated by fewer than $3 m$ elements. In this sense, our theorem is optimal.

A result similar to the coprime case of our theorem but involving a constant multiple of $d^{2} / \log d$ in place of the present $\left\lfloor\frac{3}{2} d\right\rfloor$ had been given by Fisher in [4]; the noncoprime case was also considered there. We are indebted to Professor J. D. Dixon for drawing the problem to our attention.

\section{THE COPRIME CASE}

As complete reducibility of finite linear groups is unaffected by field extensions (see $\S$ VII.1 in [8]), F can always be assumed as large as we wish.

The plan for the coprime case outlined in the Introduction directly proves the theorem with $\left\lfloor\frac{3}{2} d\right\rfloor+1$ in place of $\left\lfloor\frac{3}{2} d\right\rfloor$. The way to improve on this lies in considering transition in both directions between $G L(V)$ and $P G L(V)$, the factor group of $G L(V)$ modulo the group $Z$ of all the scalar transformations.

First, let $p$ be any prime different from the characteristic of $\mathbb{F}$, and $H$ a Sylow $p$-subgroup of $G$. Extend $F$ if necessary, to ensure that $Z$ has a finite p-subgroup $P$ which is not contained in $H$. Of course $P$ contains $H \cap Z$, so $H Z / Z \cong H P / P$. Further, $P$ is not contained in the Frattini subgroup of $H P$, and so $H P$ needs more generators than $H P / P$ does. From the theorem of Isaacs applied to $H P$, one can therefore conclude that $H Z / Z$ can be generated by $\left\lfloor\frac{3}{2} d\right\rfloor-1$ elements. The coprimality assumption ensures that this argument is available for all relevant primes, and so the theorem of Lucchini and Guralnick yields that $G Z / Z$ can be generated by $\left\lfloor\frac{3}{2} d\right\rfloor$ elements.

Next, let $M$ be a subgroup of $G$ minimal with respect to $M Z=G Z$. By a familiar argument, $M \cap Z$ is then contained in the Frattini subgroup of $M$, 
so $M /(M \cap Z) \cong M Z / Z=G Z / Z$ means that $M$ can be generated by $\left\lfloor\frac{3}{2} d\right\rfloor$ elements. Moreover, every elementary abelian quotient of $M$ is isomorphic to a quotient of $G Z / Z$, so we can further conclude that $M / M^{\prime}$ can be generated by $\left\lfloor\frac{3}{2} d\right\rfloor-1$ elements. A well-known result of Gaschütz [5] now allows us to combine these conclusions as follows: $M$ is generated by $\left\lfloor\frac{3}{2} d\right\rfloor$ elements one of which can be chosen within $M^{\prime}$. On the other hand, by Dedekind's law we have $G=M(G \cap Z)$, and of course $G \cap Z$ is cyclic. On replacing a generator of $M$ lying in $M^{\prime}$ by its product with a generator of $G \cap Z$, the $\left\lfloor\frac{3}{2} d\right\rfloor$-element generating set of $M$ becomes a generating set of $G$. This completes the proof of the coprime case.

\section{FIRST STEPS TOWARDS THE GENERAL CASE}

The proof of the general case will occupy the rest of the paper. Throughout, $\mathbb{F}$ denotes a field, $V$ an $\mathbb{F}$-space of dimension $d$, and $G$ a finite completely reducible subgroup of $G L(V)$; we also retain the convention that $Z$ stands for the centre of $G L(V)$. The coprime case having been dealt with, we now assume that the characteristic of $\mathbb{F}$ is not 0 . Standard results on changing fields (see $\S$ VII.1 in [8]) ensure that one can change first to the algebraic closure of $\mathbb{F}$, and then to any finite subfield which is a splitting field for $G$. We take advantage of this by assuming that

$\mathbb{F}$ is finite and contains all roots of $x^{\exp G}=1$

(here $\exp G$ stands for the exponent of $G$ ). For ease of expression we also assume that

$$
G \geq Z
$$

This can be done without loss of generality. Indeed, it is an elementary exercise to show that if a central product of an arbitrary finite group $G$ with a finite cyclic group $Z$ can be generated by $n$ elements, then so can $G$ itself. On the other hand, while the exponent of $G Z$ may be larger than the exponent of $G$, it is easy to see that if $\mathbb{F}$ contains all solutions of $x^{\exp G}=1$ then it also contains all solutions of $x^{\exp G Z}=1$.

Our aim in this section is to prove the theorem under the additional hypothesis that $G$ has an irreducible quasisimple normal subgroup $K$. Of course now $\mathbb{F}$ is a splitting field for $K$, so $\mathbb{C}_{G}(K)=Z$, and hence the generalized Fitting subgroup $F^{*}(G)$ is just $K Z$. (The reader is assumed to be familiar with the terminology and basic results in $\S \mathrm{X} .13$ of [8].) In particular, $F^{*}(G)$ can be generated by 2 elements. In view of the theorem of Lucchini and Guralnick, it will therefore be more than sufficient to prove the following.

Lemma. If $G$ has an irreducible quasisimple normal subgroup, then for all primes $p$ the sectional p-rank of $G / F^{*}(G)$ is at most $\left\lfloor\frac{3}{2} d\right\rfloor-3$, except when $p=2$ and $d=\left|G / F^{*}(G)\right|=2$.

Proof. Now $G / F^{*}(G)$ is isomorphic to a subgroup of Out $K$. If an automorphism of a group is trivial on the central factor group, it must fix each 
commutator: since $K$ is perfect, this makes it easy to see that in turn Out $K$ is isomorphic to a subgroup of $\operatorname{Out}(K / Z(K))$. From the description of the outer automorphism groups of the simple groups given in the Atlas [2], one readily sees that they all have sectional $p$-rank at most 3 , for all $p$. As $\left\lfloor\frac{3}{2} d\right\rfloor-3 \geq 3$ when $d \geq 4$, we need only be concerned with the cases $d=2$ and $d=3$.

All irreducible but imprimitive linear groups of dimension at most 3 are obviously soluble: hence now our $G$ is primitive. All primitive linear groups of dimension at most 3 are explicitly known, so it is just a matter of checking lists (Bloom [1], Hartley [7]). Not quite: some lists restrict attention to the unimodular case. To overcome this hurdle, consider a finite extension $\mathbb{E}$ of $\mathbb{F}$ in which each equation $x^{d}=f$ with $f \in \mathbb{F}$ has a root, and denote the centre of $G L(d, \mathbb{E})$ by $Z^{*}$ : then $G<S L(d, \mathbb{E}) Z^{*}$. Put $H=G Z^{*} \cap S L(d, \mathbb{E})$; then $K$ is a normal subgroup of $H$ so $H$ is primitive and therefore listed, while $H / F^{*}(H)$ and $G / F^{*}(G)$ are isomorphic to the same subgroup of Out $K$ and so it suffices to check on $H / F^{*}(H)$.

(In fact, one finds that $\left|G / F^{*}(G)\right| \leq d$ whenever $d \leq 3$.)

\section{IRREDUCIBLE LAYER}

The aim of this section is to prove our theorem under the assumption that the layer $E(G)$ of $G$ is irreducible.

Lemma. If $K_{1}, \ldots, K_{r}$ are the quasisimple subnormal subgroups of $G$ and if the (normal) subgroup $E$ they generate is irreducible, then the sectional p-rank of $G / F^{*}(G)$ is at most $\left\lfloor\frac{3}{2} d\right\rfloor-2 r-1+\left\lfloor\frac{r}{p}\right\rfloor$, except when $r=1, p=2$, and $d=\left|G / F^{*}(G)\right|=2$.

Proof. The case of $r=1$ is just the lemma of $\S 3$ so suppose that $r \geq 2$. As $E$ is irreducible, $\mathbb{C}_{G}(E)=Z$, and so $F^{*}(G)=E Z$. For each $i$, choose an irreducible $K_{i}$-subspace $V_{i}$, and denote by $K_{i} \downarrow V_{i}$ the restriction of $K_{i}$ in $G L\left(V_{i}\right)$. The linear span of $K_{i}$ is a subalgebra of $\operatorname{End}_{\mathbb{F}} V$; let $L_{i}$ denote the normalizer of $K_{i}$ in the group of units of that subalgebra, and $N$ the intersection of the normalizers $\mathbb{N}_{G L(V)}\left(K_{i}\right)$. Of course, $Z \leq L_{i} \leq N \geq G$. By Corollary 2 in $\S 4$ of [10], we know that $N / F^{*}(G)$ is the direct product of the $L_{i} / K_{i} Z$. Of course $F^{*}\left(L_{i}\right)=K_{i} Z$, restriction to $V_{i}$ is an (abstract) isomorphism on $L_{i}$, and the lemma of $\S 3$ is applicable with $L_{i} \downarrow V_{i}, K_{i} \downarrow V_{i}$ in the roles of $G, K$ : with $d_{i}=\operatorname{dim} V_{i}$, the conclusion is that either the sectional $p$-rank of $L_{i} / K_{i} Z$ is at most $\left\lfloor\frac{3}{2} d_{i}\right\rfloor-3$ oR $p=2$ and $d_{i}=\left|L_{i} / K_{i} Z\right|=2$. As $d_{i} \geq 2$ for all $i$ and $d=\prod d_{i} \geq \sum d_{i}$, we can conclude that the sectional $p$ rank of $N / F^{*}(G)$ is at most $\left\lfloor\frac{3}{2} d\right\rfloor-2 r-1$ except perhaps when $r=2, p=2$, and $d_{i}=\left|L_{i} / K_{i} Z\right|=2$ for $i=1,2$. The same holds then for $(G \cap N) / F^{*}(G)$ in place of $N / F^{*}(G)$.

As $\left\{K_{1}, \ldots, K_{r}\right\}$ is the set of all components of $G$, conjugation gives a permutation representation of $G$ on this set, with kernel $G \cap N$ : thus $G /(G \cap N)$ can be thought of as a permutation group of degree $r$. It follows from Kovács 
and Praeger [11] that the sectional $p$-rank of any permutation group of degree $r$ is at most $\left\lfloor\frac{r}{p}\right\rfloor$. This completes the proof of the lemma, but for the exceptional case detailed in the second last sentence of the previous paragraph. In that case $d=4$ and $p=r=2$, so what we require of $G / F^{*}(G)$ is that its sectional 2-rank be at most 2. When $K_{1}$ and $K_{2}$ are normal in $G$, this follows without further argument. If $K_{1}$ and $K_{2}$ are conjugate in $G$, then $L_{1}$ and $L_{2}$ are also $G$-conjugate and so $G N / F^{*}(G)$ is a nonabelian group of order 8: consequently, the desired conclusion is still available.

Under the hypotheses of this lemma, our theorem will now follow from the Lucchini-Guralnick theorem if we can show that $F^{*}(G)$ can be generated by $2 r-\left\lfloor\frac{r}{2}\right\rfloor$ elements. (Recall that the case $r=1$ has already been disposed of in $\S 3$.) In turn, this requires only that any direct product of $r$ nonabelian simple groups be generated by the given number of elements. By the variant of a lemma of Wiegold [14] mentioned in our $\S 1$, any such direct product can be generated by $2+\left\lceil\log _{60} r\right\rceil$ elements. Of course $2+\log _{60} r \leq r+1 \leq 2 r-\left\lfloor\frac{r}{2}\right\rfloor$ whenever $r \geq 2$, so we are done.

\section{MULTIPLICITY-FREE LAYER}

The final step in the first half of the proof of the theorem is now at hand.

Lemma. Suppose that $G$ is irreducible, that it does have quasisimple subnormal subgroups, and that the product $E$ of these is multiplicity-free (in the sense that $V$ as $\mathbb{F} E$-module is a direct sum of pairwise nonisomorphic irreducible submodules). Then $G$ can be generated by $\left\lfloor\frac{3}{2} d\right\rfloor$ elements.

Proof. Let $K_{1}, \ldots, K_{s}$ be the components of $G$, and let $V \downarrow E=V_{1} \oplus \ldots \oplus V_{t}$ with irreducible $V_{j}$. The case of $t=1$ having been dealt with in $\S 4$, assume that $t \geq 2$. Of course each $K_{i} \downarrow V_{j}$ is either trivial or quasisimple, and to each $i$ there is at least one $j$ such that $K_{i} \downarrow V_{j}$ is nontrivial. For any one $j$, let $r$ be the number of nontrivial $K_{i} \downarrow V_{j}$ : as $G$ is irreducible, the $V_{j}$ form a single $G$-orbit, so this $r$ is independent of $j$; also, by the foregoing, $s \leq r t$. This may be the point to note that each $V_{j}$ has dimension $\frac{d}{t}$.

Consider $N=\cap \mathbb{N}_{G L(V)}\left(V_{j}\right)=\bigoplus G L\left(V_{j}\right)$. With $Z_{j}$ standing for the centre of $G L\left(V_{j}\right)$, now $\mathbb{C}_{G L(V)}(E)=\bigoplus Z_{j}$, so $\mathbb{C}_{G}(E)$ is a subgroup of a direct product of $t$ cyclic groups. In particular, it follows that $\mathbb{C}_{G}(E)=F(G)$, hence $Z \leq$ $F^{*}(G)=E \mathbb{C}_{G}(E) \leq N$. Of course $G \cap N$ is a normal subgroup of $G$, so we also have $F^{*}(G)=F^{*}(G \cap N)$.

It is an elementary exercise that if $X$ is a finite subgroup of a finite direct product $\prod Y_{j}$ with coordinate projections $\pi_{j}: \prod Y_{j} \rightarrow Y_{j}$ then $F^{*}(X)=X \cap$ $\prod F^{*}\left(X \pi_{j}\right)$ and so $X / F^{*}(X)$ is isomorphic to a subgroup of $\Pi\left(X \pi_{j} / F^{*}\left(X \pi_{j}\right)\right)$. Apply this with $X=G \cap N$ and $Y_{j}=G L\left(V_{j}\right)$ to obtain that $(G \cap N) / F^{*}(G)$ is isomorphic to a subgroup of $\Pi\left(G_{j} / F^{*}\left(G_{j}\right)\right)$ where $G_{j}=(G \cap N) \downarrow V_{j}$. We know that $E \downarrow V_{j}$ is the product of $r$ elementwise commuting quasisimple groups, that it is normal in $G_{j}$, and it is irreducible. By the lemma of $\S 4$, the 
sectional $p$-rank of $G_{j} / F^{*}\left(G_{j}\right)$ is at most $\left\lfloor\frac{3}{2} \frac{d}{t}\right\rfloor-2 r-1+\left\lfloor\frac{r}{p}\right\rfloor$, except when $r=1, p=2$, and $\frac{d}{t}=\left|G_{j} / F^{*}\left(G_{j}\right)\right|=2$. Since the $G_{j}$ are quotients of $G \cap N$ modulo $G$-conjugate kernels, either all $G_{j}$ are exceptional in this sense or none of them is. When none of them is exceptional, we conclude that the sectional $p$-rank of $(G \cap N) / F^{*}(G)$ is at most $\left\lfloor\frac{3}{2} d\right\rfloor-2 r t-t+\left\lfloor\frac{r}{p}\right\rfloor t$.

On the other hand, $G \cap N$ is the kernel of the permutation representation of $G$ on the set $\left\{V_{1}, \ldots, V_{t}\right\}$, so by a previous argument the sectional $p$-rank of $G /(G \cap N)$ is at most $\left\lfloor\frac{t}{p}\right\rfloor$. It follows that, but for the exceptional case, the sectional $p$-rank of $G / F^{*}(G)$ is at most $\left\lfloor\frac{3}{2} d\right\rfloor-q$ where

$$
q=2 r t+t-\left\lfloor\frac{r}{2}\right\rfloor t-\left\lfloor\frac{t}{2}\right\rfloor \text {. }
$$

In the exceptional case, $G / F^{*}(G)$ is isomorphic to a subgroup of a (permutational or nonstandard) wreath product of a group of order 2 with the symmetric group of degree $t$, and hence also to a subgroup of the symmetric group of degree $2 t$ : thus in this case the sectional $p$-rank of $G / F^{*}(G)$ is at most $t$, for all $p$. Recall that in this case $d=2 t$ and $r=1$.

To complete the argument in the nonexceptional case on the pattern which must be familiar by now, we need to show that $F^{*}(G)$ can be generated by $q-1$ elements. We have already used special cases of Lemma 5.1 of Wiegold [13]: if a perfect group can be generated by $m$ elements and an abelian group can be generated by $n$ elements, then any central product of these two groups can be generated by $\max \{m, n\}$ elements. Now we know that $E(G)$ is the product of at most $r t$ components, so by the variant of that other lemma of Wiegold [14] in our $\S 1$, this perfect group can be generated by $2+\left\lceil\log _{60} r t\right\rceil$ elements. Further, $F(G)$ is known to be an abelian group which can be generated by $t$ elements. Of course $q-1 \geq t$ always holds and, as $t \geq 2$, so does $q-1 \geq t+1 \geq 2+\left\lceil\log _{60} r t\right\rceil$ when $r=1$. If also $r \geq 2$, then $r t \geq r+t$ and hence

$$
\begin{aligned}
q-1 & \geq \frac{3}{2} r t-\frac{t}{2}-1 \\
& \geq \frac{3}{2} r+t-1 \\
& \geq 2+(r-1)+(t-1) \\
& \geq 2+\left\lceil\log _{60} r\right\rceil+\left\lceil\log _{60} t\right\rceil \\
& \geq 2+\left\lceil\log _{60} r t\right\rceil .
\end{aligned}
$$

To complete the argument in the exceptional case on the same pattern is much easier. One is required to show that each of $E(G)$ and $F(G)$ can be generated by $2 t-1$ elements: but $2 t-1 \geq t$ and $2 t-1 \geq 2+\log _{60} t$ are obvious when $t \geq 2$.

\section{THE INDUCTION}

We are now ready to prove the theorem in full generality, by induction on $d$. The assumption that $\mathbb{F}$ is a finite splitting field for all subgroups of $G$ will be maintained; the characteristic of $\mathbb{F}$ will be denoted by $p$. The initial case 
$d=1$ is obvious. The inductive hypothesis is that $d>1$ and the theorem holds in all smaller dimensions.

Case $1 . G$ is reducible.

Now $V=U \oplus W$ with $\mathbb{F} G$-submodules $U, W$ of dimension less than $d$. By the inductive hypothesis, $G \downarrow U$ and $\mathbb{C}_{G}(U) \downarrow W$ can be generated by $\left\lfloor\frac{3}{2} \operatorname{dim} U\right\rfloor$ and $\left\lfloor\frac{3}{2} \operatorname{dim} W\right\rfloor$ elements, respectively. On the other hand, $G / \mathbb{C}_{G}(U) \cong G \downarrow U$ and $\mathbb{C}_{G}(U) \cong \mathbb{C}_{G}(U) \downarrow W$, so it follows that $G$ can be generated by $\left\lfloor\frac{3}{2} d\right\rfloor$ elements.

Case 2. $G$ is irreducible and has a nonabelian normal subgroup $H$ which is not multiplicity-free.

Let $t$ denote the number of the isomorphism types of the irreducible direct summands of $V$ as $\mathbb{F} H$-module; by Clifford's theorem, these summands have a common dimension, $e$ say, and a common multiplicity, $m$ say. Then $d=$ emt, and $\mathbb{C}_{G L(V)}(H)$ is the direct sum of $t$ copies of $G L(m, \mathbb{F})$. Being a subgroup of this direct sum, $\mathbb{C}_{G}(H)$ has a faithful representation of dimension $m t$ over $\mathbb{F}$. Since $\mathbb{O}_{p}\left(\mathbb{C}_{G}(H)\right)=1$, it follows that $\mathbb{C}_{G}(H)$ has also a completely reducible faithful representation of this dimension. As $H$ is nonabelian, $e \geq 2$ : thus by the inductive hypothesis $\mathbb{C}_{G}(H)$ can be generated by $\left\lfloor\frac{3}{2} m t\right\rfloor$ elements.

On the other hand, $H$ has a faithful completely reducible and multiplicityfree representation over $\mathbb{F}$, whose dimension is et and whose equivalence type is $G$-invariant: let $\rho: H \rightarrow G L(U)$ be such a representation. To each $g$ in $G$ there exist elements $\bar{g}$ in $G L(U)$ such that $\left(g^{-1} h g\right) \rho=\bar{g}^{-1}(h \rho) \bar{g}$ for all $h$ in $H$. In fact, the $\bar{g}$ with this property form a right coset module $\mathbb{C}_{G L(U)}(H \rho)$. The union of these cosets is a group $\bar{G}$ which is finite (because $\mathbb{F}$ is) and completely reducible (because now each $H$-admissible subspace of $U$ has a unique $H$-admissible complement, and thus if this subspace admits $\bar{G}$, so does that complement). By our assumption, $m \geq 2$ : so by the inductive hypothesis $\bar{G}$ can be generated by $\left\lfloor\frac{3}{2}\right.$ et $\rfloor$ elements. As $\bar{G} / \mathbb{C}_{G L(U)}(H \rho) \cong G / \mathbb{C}_{G}(H)$, it follows that $G$ can be generated by $\left\lfloor\frac{3}{2} m t\right\rfloor+\left\lfloor\frac{3}{2} e t\right\rfloor$ elements. Since $e \geq 2$ and $m \geq 2$, one has $e m \geq e+m$, so this is good enough.

Case 3. $G$ is irreducible and $\mathbb{C}_{G}(F(G)) \leq F(G)$.

Let $P$ be a Sylow $p$-subgroup of $G$, and set $H=P F(G)$. Since $G$ is irreducible, $\mathbb{O}_{p}(G)=1$, and so $P \cap F(G)=1$. Because $\mathbb{C}_{G}(F(G)) \leq F(G)$, it follows that $\mathbb{O}_{p}(H)=1$; therefore $H$ has a completely reducible faithful representation of dimension $d$ over $\mathbb{F}$. As $H$ is soluble, the Fong-Swan-Rukolaine theorem (22.1 in [3]) now gives that one can also view $H$ as a subgroup of $G L(d, \overline{\mathbb{F}})$ for a suitable field $\overline{\mathbb{F}}$ of characteristic 0 . Then $P \cap Z(H)=1$ shows that the natural map to $P G L(d, \overline{\mathbb{F}})$ is one-to-one on $P$. By the argument in the first half of the proof of the coprime case, $P$ can therefore be generated by $\left\lfloor\frac{3}{2} d\right\rfloor-1$ elements. Of course for Sylow subgroups of $G$ corresponding to other primes, that argument is available already over $\mathbb{F}$. The second half of that proof made no use of the coprimality assumption, so it can be used to finish off this case as well. 


\section{THE REMAINING CASE}

In the remaining case, $G$ is irreducible and the layer $E(G)$ of $G$ is nontrivial and multiplicity-free: so that is just the case dealt with in the lemma of $\S 5$.

\section{REFERENCES}

1. D. M. Bloom, The subgroups of $P S L_{3}(q)$ for odd $q$, Trans. Amer. Math. Soc. 127 (1967), 150-178.

2. J. H. Conway, R. T. Curtis, S. P. Norton, R. A. Parker, and R. A. Wilson, Atlas of finite groups, Clarendon Press, Oxford, 1985.

3. Charles W. Curtis and Irving Reiner, Methods of representation theory I, Wiley, New York and Chichester Brisbane, Toronto, 1981.

4. R. K. Fisher, The number of generators of finite linear groups, Bull. London Math. Soc. 6 (1974), 10-12.

5. Wolfgang Gaschütz, Zu einem von B. H. und H. Neumann gestellten Problem, Math. Nachr. 14 (1955), 249-252 (1956).

6. Robert M. Guralnick, On the number of generators of a finite group, Arch. Math. 53 (1989), 521-523.

7. R. W. Hartley, Determination of the ternary collineation groups whose coefficients lie in $G F\left(2^{n}\right)$, Ann. of Math. (2) 27 (1925), 140-158.

8. B. Huppert and N. Blackburn, Finite groups II, III, Springer-Verlag, Berlin, Heidelberg, and New York, 1982.

9. I. M. Isaacs, The number of generators of a linear p-group, Canad. J. Math 24 (1972), 851-858.

10. L. G. Kovács, On tensor induction of group representations, J. Austral. Math. Soc. Ser. A (to appear).

11. L. G. Kovács and Cheryl E. Praeger, Finite permutation groups with large abelian quotients, Pacific J. Math. 136 (1989), 283-292.

12. Andrea Lucchini, $A$ bound on the number of generators of a finite group, Arch. Math. $\mathbf{5 3}$ (1989), 313-317.

13. James Wiegold, Growth sequences of finite groups, J. Austral. Math. Soc. 17 (1974), 133-141.

14. $\_$, Growth sequences of finite groups III, J. Austral. Math. Soc. Ser. A 25 (1978), 142-144.

Mathematics IAS, Australian National University, GPO Box 4, Canberra 2601, Australia

Department of Mathematics, University of Florida, Gainesville, Florida 32611 